\title{
Analysis of the Electronic Structure, Hyperfine Structure, and Volume Isotope Shifts in the Low Lying States of Ba I and Ba II
}

\author{
T. Olsson and A. Rosén
}

Department of Physics, Chalmers University of Technology, University of Göteborg, S-412 96 Göteborg, Sweden

and

B. Fricke and G. Torbohm

Department of Physics, University of Kassel, D-3500 Kassel, Germany

Received July 31, 1987; accepted December 18, 1987

\begin{abstract}
Relativistic multi-configuration Dirac Fock (MCDF) wavefunctions coupled to good angular momentum $J$ have been calculated for low lying states of $\mathrm{Ba} \mathrm{I}$ and $\mathrm{Ba}$ II. These wavefunctions are compared with semiempirical ones derived from experimental atomic energy levels. It is found that significantly better agreement is obtained when close configurations are included in the MCDF wavefunctions. Calculations of the electronic part of the field isotope shift lead to very good agreement with electronic factors derived from experimental data. Furthermore, the slopes of the lines in a King plot analysis of many of the optical lines are predicted accurately by these calculations. However, the MCDF wavefunctions seem not to be of sufficient accuracy to give agreement with the experimental magnetic dipole and electric quadrupole hyperfine structure constants.
\end{abstract}

\section{Introduction}

One of the goals of the fundamental research in atomic physics is to understand the various inter-electronic and nuclear-electronic interactions in complicated atoms. The reason for the contribution of various terms is the electromagnetic interaction itself, which is only understood in terms of a perturbation expansion in quantum electrodynamics (QED), where the first term is the electric $1 / r$ (Coulomb-) interaction and the next are the magnetic, vacuum-polarization, self-energy terms, etc. This easily makes clear why a large number of terms in the electron-electron as well as the electron-nuclear interaction appear in the theoretical description. The ever increasing accuracy of new laser spectroscopic methods [1-3] is so good that a large number of the theoretical terms can be tested experimentally. In this paper we discuss the terms due to the coupling of the electronic cloud with the nucleus usually referred to as the hyperfine structure (hfs) and the isotope shift (IS).

The IS can be divided into the field shift caused by the difference of the finite extension of the nuclei and the mass shift, originating from the difference in the finite mass of the nuclei [4-10] under consideration. The hfs $[4,11]$, as well as the IS, can be expressed as a sum of products of electronic and nuclear quantities, implying that nuclear properties can only be evaluated from experimental data if the electronic part of each interaction is known from other experiments or calculations. For evaluation of electronic properties the inverse holds, i.e., the nuclear properties have to be known from independent, semi-empirical analysis or from ab initio calculations. Generally, a deeper insight into the interactions and into the power of different theoretical methods can be achieved by comparing theoretical ab initio, and semi-empirical values of as many properties as possible with the corresponding experimental data.

For every type of $a b$ initio calculation one needs good wavefunctions, which can be obtained by solving either the non-relativistic Schrödinger or the relativistic Dirac equations. In the usually used approximations such as the independent particle and the central-field models [12,13] with the $1 / r$ Coulomb potential one gets the Hartree-Fock (HF) [14] or Dirac-Fock (DF) $[15,16]$ method. The remaining part of the electrostatic and magnetic interactions in the atoms can then be treated by many-body perturbation theory (MBPT) to a certain order $[17,18]$. In such an approach, higher order wavefunctions are calculated by including one-, two- and three-particle excitations of the zero order wavefunction. This zero order wavefunction is normally evaluated for the average energy of the different terms within the actual configuration. That approach is in general a good approximation for systems with one open shell. However, the electronic wavefunctions for the terms obtained for two open shells are in many cases so different from each other that an $L S$ dependent $\mathrm{HF}$ - or $L S J$-dependent DF treatment is more appropriate in calculating the zero order wavefunction.

An alternative to MBPT is the Multi-Configuration Hartree-Fock (MCHF) [19] or in the relativistic case the Multi-Configuration Dirac-Fock (MCDF) [20, 21] method. The wavefunction for the state under consideration is in this approach expanded as a linear combination of determinants with the same total symmetry, i.e., the same parity, total orbital- and spin angular momentum in the nonrelativistic treatment or the same total angular momentum $J$ in the relativistic treatment. In all these approaches the coefficients and the wavefunctions are determined in a self-consistent procedure without any use of experimental quantities.

A quite different and commonly used method, in the analysis of different types of structure in the atom, is of a semi-empirical nature in which parametrized expressions are fitted to experimental quantities. Historically, the Rydberg formula for hydrogen-like systems was extended to other systems like the alkali atoms by introducing effective quantum numbers $n_{\text {eff }}$ instead of the principal quantum number $n$. Other examples are the use of the direct- and exchangeelectrostatic integrals [12], Trees parameters [17, 22] and spin-orbit parameters to determine so called intermediate coupled wavefunctions. In recent years the semi-empirical 
Multi-Channel-Quantum Defect Theory (MCQDT) [23, 24] has been used with great success in the analysis of Rydberg series of two electron systems by especially Aymar et al. [25]. The effective parameters introduced in these methods can today be analyzed by MBPT [17] or MCHF-methods [26].

In order to test these theoretical methods the interest of many groups has now focused on two electron systems and in particular the alkaline earth elements [27]. A MBPT treatment is here more difficult to perform than in the alkali atoms due to the correlation between the valence electrons although some non-relativistic calculations have recently been performed $[28,29]$. It is for these systems important to calculate $L S$-dependent zero order wavefunctions as the starting of the MBPT approach. For heavy atoms such an approach leads to the evaluation of $L S J$-dependent wavefunctions, in which the effect of correlation and relativity is treated on an equal basis.

Recently, we presented some relativistic MCDF calculations as a step towards more sophisticated calculations for the interpretation of IS. The resulting wavefunctions were used to calculate the charge density at the nucleus for low lying states of $\mathrm{Ba}$ I and $\mathrm{Ba}$ II [30] and in the $n s^{2}{ }^{2} S_{0}$, nsnp ${ }^{1} P_{1}$ and ${ }^{3} P_{1}$ states of other group IIa and IIb elements [31] and $\mathrm{Au}$ I [32]. The calculations accurately reproduced the optical volume isotope shifts.

In addition to the experimental work analyzed at the time of our earlier calculations [30] new values of isotope shifts in $\mathrm{Ba} \mathrm{I}$ and $\mathrm{Ba}$ II relative to the ionic ground state have been derived by following the $6 s n l$ Rydberg series to highly excited states [33, 34]. Furthermore, there exists now IS-measurements in many spectral lines between low lying states in the Ba atom and ion [35-40]. These new experimental data can be used to extract information about the relative magnitude of the charge density at the nucleus in a number of states in Ba I and Ba II. An extensive comparison between $a b$ initio and experimental charge density differences at the nucleus is therefore possible. This motivated an extension of our earlier calculations for $\mathrm{Ba}$ to other configurations. In addition we present a comparison between the square of the expansion coefficients of the wavefunctions obtained from the MCDF calculations and obtained from a semi-empirical analysis in which parametrized expressions are fitted to experimental energy levels.

A more critical test of the calculations is to compare other properties such as the magnetic dipole and electric quadrupole hyperfine interaction constants. These quantities have different radial and angular dependences than the volume isotope shift and represent another test of the accuracy of the wavefunctions outside the nucleus. The $a b$ initio MCDF wavefunctions have therefore been used for evaluation of $a b$ initio values for the magnetic-dipole and electric quadrupole interaction constants for comparison with available experimental values.

A short review of the theoretical background is given in the next section. Results and a comparison with different experimental quantities are presented in Section III with a summary and conclusion in Section IV.

\section{Theory}

\subsection{Computational method}

The relativistic treatment of the atom in this work is based on the solution of the Dirac equation within the MultiConfiguration Dirac-Fock (MCDF) approach [20, 21, 42, 43]. In this treatment the Hamiltonian for the many electron system is written as usual

$$
H_{0}=\sum_{i} h_{i}+\sum_{i<j} V_{i j}^{c}
$$

with

$h_{i}=c \boldsymbol{\alpha}_{i} \cdot \boldsymbol{p}_{i}+m c^{2}\left(\beta_{i}-1\right)-V_{i}^{N}$

and

$$
V_{i j}^{c}=\frac{e^{2}}{4 \pi \varepsilon_{0} r_{i j}}
$$

where $V^{c}$ is the electron-electron interaction and $V^{N}$ is the monpole part of the electron-nucleus Coulomb interaction. The ansatz for the wavefunction is a superposition of configuration state functions $|C S F\rangle$ representing states with a certain angular momentum $J$ and parity.

$\Psi=\sum_{n} c_{n}|\mathrm{CSF}\rangle_{n}$

The configuration state functions are constructed from relativistic single particle wavefunctions as described for example in Refs. [42 and 43]. All interactions considered in addition to $H_{0}$ are collected in the Hamiltonian

$H_{1}=h_{\mathrm{B}}+h_{\mathrm{QED}}+h_{\mathrm{hfs}}+h_{\mathrm{IS}}$

where the summation over $i$ and $j$ is understood to be included. $h_{\mathrm{B}}$ is the Breit-operator [44], $h_{\mathrm{QED}}$ contains the Quantum electrodynamic corrections, $h_{\text {hrs }}$ the hyperfine structure and $h_{1 \mathrm{~S}}$ the isotope shift operator. These interactions are too compliccated to be taken into account directly in the Hamiltonian $H_{0}$ and thus are usually not included in the self-consistency procedure used to determine the eigenvalues, wavefunctions and total energy for the atomic system in the MCDF method. Their influence on the total energy and energy eigenvalues are only evaluated within first-order perturbation theory by use of the wavefunctions of the zeroorder Hamiltonian [45-51].

As a result of a MCDF procedure with the ansatz acc. to Eqn. (3) all these states with good $J$ are mixed and the expansion coefficients $c_{i}$ as well as the one-particle functions $\phi$ are determined to self-consistency. In adding the Breit and QED corrections by first-order perturbation theory the total energy for the particular state is obtained.

The effect of an extended nucleus is included by use of a two-parameter Fermi-nuclear charge distribution [52]. The two parameters are $R_{\mathrm{c}}$, the half-charge radius, and $t$ the thickness parameter. Of course the choice of a specific set of nuclear parameters affects the total energy of the atom very drastically. However, as long as the choice of nuclear parameters is not too far from reality this does not play any important role for the electronic factor or term splittings which are given as differences of large numbers. Following Shera et al. [53] we have chosen the parameters $R_{\mathrm{c}}=5.763 \mathrm{fm}$ and $t=2.17 \mathrm{fm}$ in all the calculations. These values are somewhat different than with the values used in the earlier calculations [31] which were taken from the liquid drop model [54].

\subsection{Isotope Shift Theory}

Detailed studies of optical spectral lines of electronic and 
muonic $X$-rays from a sample of different isotopes shows that there exist a shift in the center of gravity between the different isotopes. This shift, which is known as the isotope shift (IS), is given as the sum of the volume (field) isotope shift $\delta v_{\mathrm{FS}}$ and the mass shift which can be divided into the normal $\delta v_{\mathrm{NMS}}$ and the specific mass shift $\delta v_{\mathrm{SMS}}[4-10]$. In a certain transition the $\delta v_{\mathrm{NMS}}$ can be evaluated exactly while some semi-empirical procedure is usually used in the estimate of the $\delta v_{\mathrm{SMS}}$.

We will in this work limit ourselves to the volume shift $\delta v_{\mathrm{FS}}$. The origin of this shift is due to the penetration of the electrons in the atom into the atomic nucleus. The energy contribution for a certain isotope $A_{1}$ in an atomic state $e_{u}$ due to this penetration is given as

$E_{e_{u}}^{A_{1}}=-e^{2} \iint d r d r^{\prime} \frac{1}{\left|r-r^{\prime}\right|} \varrho_{A_{1}}(\boldsymbol{r}) \varrho_{e_{u}}\left(\boldsymbol{r}^{\prime}\right)$

where $\varrho_{A_{1}}$ is the nuclear charge distribution for isotope $A_{1}$ and $\varrho_{e_{u}}$ is the electronic charge distribution. This will give the following relation for the FS of two isotopes 1 and 2 in a transition between two states $u$ and $t$

$\delta v_{\mathrm{FS}, u t}^{A_{1} A_{2}}=E_{e_{u}}^{A_{1}}-E_{e_{u}}^{A_{2}}-E_{e_{t}}^{A_{1}}+E_{e_{t}}^{A_{2}}$

which can be evaluated directly or by perturbation theory. Following the procedure in the earlier work [31] the electron density within the nucleus i.e., $r<R$ is expressed as a polynomial

$\varrho=a_{0}+a_{2} r^{2}+a_{4} r^{4}+\ldots$

and the final shift is usually written as

$\delta v_{\mathrm{FS}, \text { ut }}^{A_{1} A_{2}}=F \Delta\left\langle r^{2}\right\rangle^{A_{1} A_{2}} K$

where $F$ is the electronic factor which is proportional to the electron density difference in the two configurations at $r=0$,

$F=\frac{2 \pi}{3} Z e^{2} \Delta|\Psi(0)|^{2}$

and $K$ is the so called nuclear parameter which takes into account the variation of the electronic charge density over the nuclear volume. (The higher polynomials of the expression of $\varrho$ ). Detailed analysis of this factor in the earlier work [31] showed that it was more or less independent of the investigated optical transition and only a function of the atomic number $Z$, with a value of $K=0.97$ for $\mathrm{Ba}$.

Interesting information about the volume isotope shifts in different transitions can be obtained in a King plot analysis $[10,55]$. In this graphical analysis the experimental IS corrected for the NMS in one transition $i$ is plotted vs the IS in another transition $j$ known as the reference transition in the following way

$$
\begin{aligned}
\frac{m_{x y}}{m_{12}} \delta v_{i}^{A_{1} A_{2}}= & \frac{F_{i}}{F_{j}} \frac{m_{x y}}{m_{12}} \delta v_{j}^{A_{1} A_{2}} \\
& +\frac{m_{x y}}{m_{12}}\left[\mathrm{SMS}_{i}^{A_{1} A_{2}}-\frac{F_{i}}{F_{j}} \mathrm{SMS}_{j}^{A_{1} A_{2}}\right]
\end{aligned}
$$

where

$m_{12}=\frac{m_{1}-m_{2}}{m_{1} m_{2}}$

and $x y$ is referring to a reference isotope pair. The slope in this line is expressed as

$k_{i}=\frac{F_{i}}{F_{j}}=\frac{\Delta \varrho_{i}}{\Delta \varrho_{j}}$ which respresents the ratio of the change of the electron density at the nucleus in the two transitions. The slopes are experimentally derived quantities which can be used for a direct test of theoretical $a b$ initio $F_{i}$ values. Furthermore, King plot analysis of transitions between different states provides a detailed $J$-dependent analysis of the charge density at the nucleus to be further exploited in Section III.

\subsection{Hyperfine structure theory}

Hyperfine structure in the atomic system is included if the momentum operator $\boldsymbol{p}_{i}$ in the Dirac Hamiltonian $h_{i}$ in eq. (2) is replaced by the generalized momentum $\left(p_{i}+e A\right)$ and the monopole part of the extended nuclear potential $V^{N}$ is replaced by $-e \phi$ so that $[11,16,56]$ :

$h_{i}+h_{\mathrm{hss} ; i}=c \alpha\left(\boldsymbol{p}_{i}+e \boldsymbol{A}\right)+m c^{2}\left(\beta_{i}-1\right)-e \Phi$

Here $\boldsymbol{A}$ is the vector potential due to the nucleus, $\Phi$ is the electrostatic potential due to the extended nucleus and $e$ is the electronic charge.

The perturbations due to the magnetic $h_{\mathrm{hfs}}^{\mathrm{M}}$ and electric $h_{\mathrm{hfs}}^{\mathrm{E}}$ hyperfine interaction are then represented by the following Hamiltonians

$h_{\mathrm{hfs}}^{\mathrm{M}}=e c \alpha \cdot A$
$h_{\mathrm{h} f \mathrm{E}}^{\mathrm{E}}=V^{N}-e \Phi$

A multipole expansion of these operators to a certain order $k$ shows that the hyperfine hamiltonian $h_{\mathrm{hfs}}=h_{\mathrm{hfs}}^{\mathrm{M}}+$ $h_{\mathrm{hfs}}^{\mathrm{E}}$ can be written as a product of spherical tensor operators acting in the nuclear $\boldsymbol{M}^{k}$ and electronic space $\boldsymbol{T}^{k}$, respectively, i.e.,

$h_{\mathrm{hrs}}=\sum_{k} \boldsymbol{T}^{k} \cdot \boldsymbol{M}^{k}$

where odd $k$ corresponds to the magnetic interaction and even $k$ to the electric interaction.

The generalized hyperfine constant of order $k$ in a certain state $J$ with magnetic quantum number $M_{J}=J$ is defined as [56]

$A_{k}(\gamma J)=\left\langle\Psi_{J J}\left|T_{0}^{k}\right| \Psi_{J J}\right\rangle M_{0}^{k}$

where $M_{0}^{k}$ are the generalized nuclear moments, which are related to the commonly used magnetic dipole moment $\mu_{1}$ and electric quadrupole moment $Q$ in the following way.

$M_{0}^{1}=\mu_{1} I, \quad M_{0}^{2}=\frac{e}{2} Q$.

The commonly used $a$ and $b$ factors are related to $A_{k}(\gamma J)$ by

$A_{1}(\gamma J)=a(\gamma J) I J ; A_{2}(\gamma J)=b(\gamma J) / 4$

$T_{0}^{k}$ are given as sum of one-electron operators as defined by Eq. 60 and 61 in Ref. 16. Using the MCDF wavefunction for $\Psi_{J J}$ according to eq. (3) one finally ends up with the following expression for the hfs-constants in a state $\gamma J$

$A_{K}(\gamma J)=M_{0}^{k} \sum_{n, p} c_{n} c_{p}\left\langle\{\mathrm{CSF}\}_{n}\left|T_{0}^{k}\right|\{\mathrm{CSF}\}_{p}\right\rangle$.

where $c_{n}$ and $c_{p}$ are the expansion coefficients of the MCDF wavefunction defined in eq. (3). In the calculation of the matrix-elements of the CSF functions one needs to evaluate the reduced matrix elements of the one-electron hyperfine 
operators, which can for the magnetic part be written as [16]

$$
\begin{aligned}
& \left\langle n \kappa|| t^{k}|| n \kappa^{\prime}\right\rangle=-2 \frac{\mu_{0}}{4 \pi} \frac{\mu_{\mathrm{B}}}{\alpha a_{0}} \frac{\kappa+\kappa^{\prime}}{k} \\
& \times\left\langle\kappa|| C^{k}||-\kappa^{\prime}\right\rangle\left[n \kappa\left|r^{-k-1}\right| n \kappa^{\prime}\right]^{\mathrm{M}}, k \text { odd. }
\end{aligned}
$$

where

$\left[n \kappa\left|r^{-k-1}\right| n \kappa^{\prime}\right]^{\mathrm{M}}=\int\left[P_{n \kappa}(r) Q_{n \kappa^{\prime}}(r)+P_{n \kappa^{\prime}}(r) Q_{n \kappa}(r)\right] r^{-k-1} \mathrm{~d} r$.

The corresponding reduced matrix element for the quadrupole interaction can be written as

$$
\begin{aligned}
& \left\langle n \kappa|| t^{k}|| n \kappa^{\prime}\right\rangle=-\frac{e}{4 \pi \varepsilon_{0}} \\
& \times\left\langle\kappa|| C^{k}|| \kappa^{\prime}\right\rangle\left[n \kappa\left|r^{-k-1}\right| n \kappa^{\prime}\right]^{\mathrm{E}}, \quad k \text { even, }
\end{aligned}
$$

where

$\left[n \kappa\left|r^{-k-1}\right| n \kappa^{\prime}\right]^{\mathrm{E}}=\int\left[P_{n \kappa}(r) P_{n \kappa^{\prime}}(r)+Q_{n \kappa}(r) Q_{n \kappa^{\prime}}(r)\right] r^{-k-1} \mathrm{~d} r$.

Using these expressions and the MCDF wavefunctions the $a(\gamma J)$ and $b(\gamma J)$ factors for the various states can be calculated.

\section{Results and discussion}

\subsection{Electronic structure for $B a I$ and $B a I I$}

The electronic structure of $\mathrm{Ba} I$ is in the ground state characterized by a closed $6 s^{2}$ configuration. Excitation of one of these $6 s$ electrons to higher states gives simple two electron systems with, in some cases, quite different character of the wavefunctions in the singlet and triplet states. Relativistic-

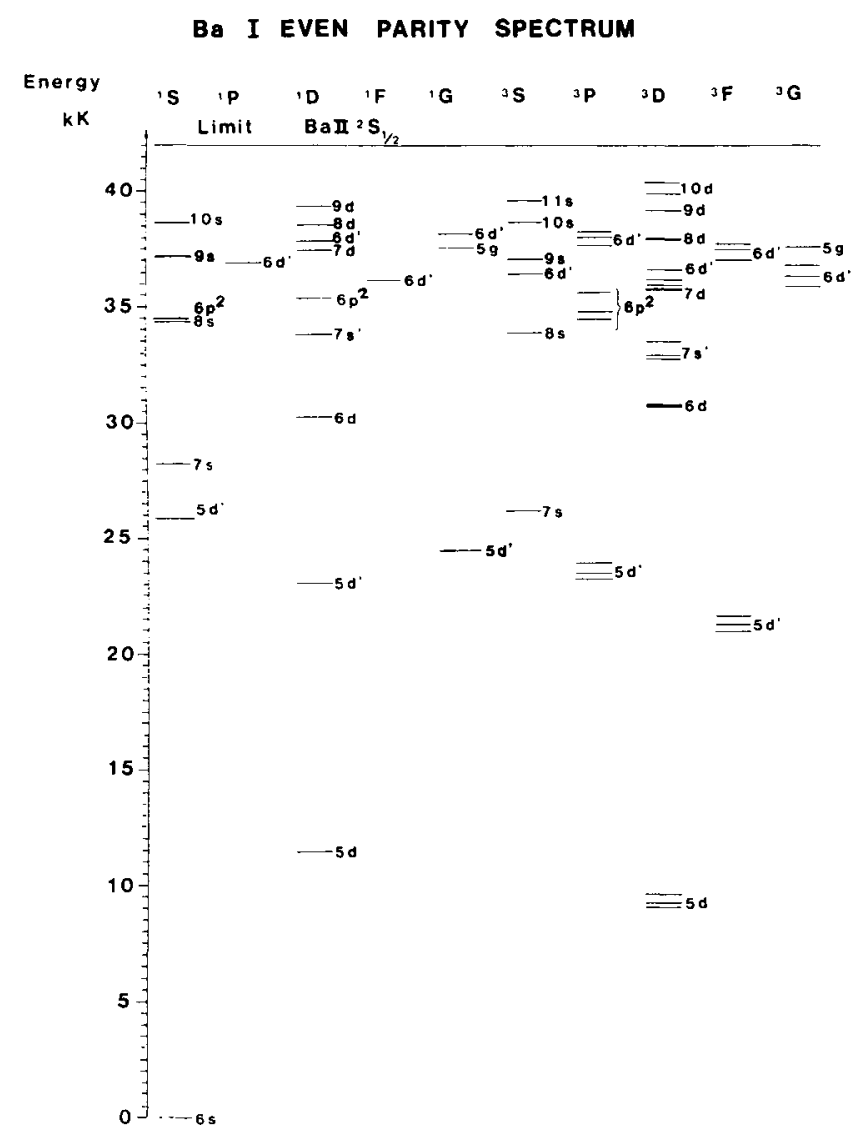

Fig. 1. (a) Schematic energy level diagram for the states of even parity in Ba I. States denoted with a prime indicates that the second electron is given as $5 d$ instead of $6 s$. The only exception are states within the $6 p^{2}$ configuration which are denoted explicitly. ally, we can only talk about the parity of a configuration and the total $J$-quantum number obtained in the coupling of the one-electron angular momenta.

A schematic experimental energy level diagram of the low lying states in $\mathrm{Ba}$ I constructed from the energy levels of Moore [58] and complementary data from more recent works [59-61] is shown in Fig. 1(a) for the even parity states and in Fig. 1(b) for the odd parity states. We notice how the states of odd parity within the two-electron excited $6 p 5 d$ configuration are close in energy with states within the $6 s 6 p$ and even more the $6 s 7 p$ configuration and are therefore expected to interact. Similarly, the even parity states within the two electron excited configurations $6 p^{2}$ and $5 d^{2}$ are expected to interact with states within the $6 s 5 d$ and $6 s^{2}$ configurations.

The relativistic MCDF calculations reported here included the following lowest configurations of positive parity; $6 s_{1 / 2}^{2}$, $6 p_{1 / 2}^{2}, 6 p_{1 / 2} 6 p_{3 / 2}, 6 p_{3 / 2}^{2}, 6 s_{1 / 2} 5 d_{3 / 2}, 6 s_{1 / 2} 5 d_{5 / 2}, 5 d_{3 / 2}^{2}, 5 d_{3 / 2} 5 d_{5 / 2}$ and $5 d_{5 / 2}^{2}$ and the lowest configurations of negative parity; $6 s_{1 / 2} 6 p_{1 / 2}$, $6 s_{1 / 2} 6 p_{3 / 2}, 6 p_{1 / 2} 5 d_{3 / 2}, 6 p_{1 / 2} 5 d_{5 / 2}, 6 p_{3 / 2} 5 d_{3 / 2}, 6 p_{3 / 2} 5 d_{5 / 2}, 6 s_{1 / 2} 4 f_{5 / 2}$ and $6 s_{1 / 2} 4 f_{7 / 2}$. In all these configurations the one-electron angular momenta were coupled to a good total angular momentum $J$. Contributions from the $6 s 4 f$ configurations were only included in the states with $J=3$ and $J=4$.

A summary of the results of the MCDF calculations for $\mathrm{Ba} I$ is presented for the even states in Table I and for the odd states in Table II. These tables are organized as follows: After the definition of the configuration and the specific state, each row represents a different calculation where DF means a single configuration and MCDF a multiconfiguration calculation. The next columns specify the square of the expansion coefficients of the configurations included in the calculations.

\section{Ba I ODD PARITY SPECTRUM}

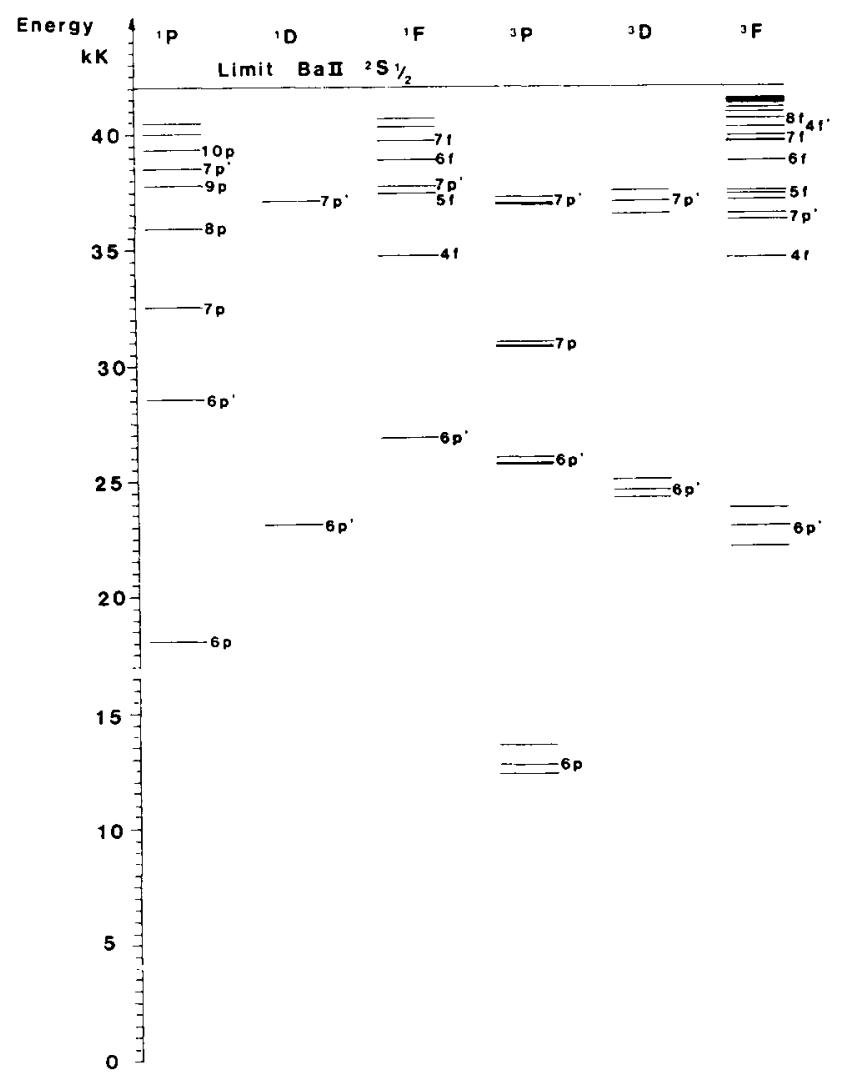

Fig. I. (b) Schematic energy level diagram for the states of odd parity in $\mathrm{Ba}$ I. States denoted with a prime indicates that the second electron is given as $5 d$ instead of $6 s$. 
Table I. A comparison of theoretical and experimental mixing of states (in percent) for the even parity configurations $6 s^{2}$ and 655 . The total energy and charge density at the nucleus is given in the last columns

\begin{tabular}{|c|c|c|c|c|c|c|c|c|c|c|c|c|c|}
\hline Config. & "State" & Method & $6 s_{1 / 2}^{2}$ & $6 p_{1 / 2}^{2}$ & $6 p_{3 / 2}^{2}$ & $6 p_{1 / 2} 6 p_{3 / 2}$ & $5 d_{3 / 2}^{2}$ & $5 d_{5 / 2}^{2}$ & $5 d_{3 / 2} 5 d_{5 / 2}$ & $6 s 5 d_{3 / 2}$ & $6 s 5 d_{s / 2}$ & $\begin{array}{l}\text { Total energy } \\
\text { (a.u.) }\end{array}$ & $\begin{array}{l}4 \pi|\psi(0)|_{\text {tot }}^{2} \\
\text { (a.u.) }\end{array}$ \\
\hline $6 s^{2}$ & ${ }^{\mathrm{t}} S_{0}$ & $\begin{array}{l}\text { DF } \\
\text { MCDF }\end{array}$ & $\begin{array}{l}100 \\
91.84\end{array}$ & 2.78 & 4.44 & & 0.34 & 0.60 & & & & $\begin{array}{l}8129.100 \\
8129.123\end{array}$ & $\begin{array}{l}4045914.2 \\
4045892.8\end{array}$ \\
\hline \multirow[t]{4}{*}{$6 s 5 d$} & ${ }^{\prime} D_{2}$ & $\begin{array}{l}\text { DF } \\
\text { MCDF } \\
\text { LS } \\
\text { Exp }\end{array}$ & & & 2.77 & 6.67 & 1.42 & 2.47 & 1.12 & $\begin{array}{l}36.27 \\
23.40 \\
40.00 \\
26.70\end{array}$ & $\begin{array}{l}63.73 \\
62.16 \\
60.00 \\
73.30\end{array}$ & $\begin{array}{l}8129.042 \\
8129.061\end{array}$ & $\begin{array}{l}4045693.2 \\
4045710.6\end{array}$ \\
\hline & ${ }^{3} \mathrm{D}_{2}$ & $\begin{array}{l}\text { DF } \\
\text { MCDF } \\
\text { LS } \\
\text { Exp }\end{array}$ & & & 0.02 & 0.11 & 0.02 & 0.04 & 0.01 & $\begin{array}{l}62.99 \\
69.94 \\
60.00 \\
73.30\end{array}$ & $\begin{array}{l}37.01 \\
29.87 \\
40.00 \\
26.70\end{array}$ & $\begin{array}{l}8129.068 \\
8129.068\end{array}$ & $\begin{array}{l}4045740.5 \\
4045739.2\end{array}$ \\
\hline & ${ }^{3} D_{3}$ & $\begin{array}{l}\text { DF } \\
\text { MCDF }\end{array}$ & & & & & & & & & 100 & 8129.067 & 4045743.4 \\
\hline & ${ }^{3} D_{1}$ & $\begin{array}{l}\mathrm{DF} \\
\mathrm{MCDF}\end{array}$ & & & & & & & & 100 & & 8129.069 & 4045738.9 \\
\hline
\end{tabular}

This clearly demonstrates the relative importance of the various configurations. The row specified by $L S$ gives the weight for the pure $L S$ state transformed into the $j j$ states presented here.

A test of these $a b$ initio calculations can be achieved by a comparison with those weights which were derived from the atomic energy levels in a semi-empirical approach as recently used by Olsson et al. $[28,62]$ for low configurations of group IIa elements. In this approach the energies for the states within a certain configuration are given in terms of electrostatic and magnetic parameters, which are treated as effective parameters in a least-square-fit to the experimental energy levels. The square of the expansion coefficients determined in this semi-empirical analysis is denoted by "Exp" and presented for some of the states in Table I and II. We notice how the weights of the configurations in the MCDF calculations often are between the pure $L S$ and semi-empirical values. The disagreement can be understood from the fact that the semiempirical expansion coefficients include contributions from other configurations in an effective way, while the MCDF wavefunctions only take into account contributions from those configurations expected to be most dominant. Another reason for the discrepancy may be that the MCDF wavefunctions do not include the Breit- and QED effects since these contributions are only added to the total energy by first-order perturbation theory.
In the last two columns of Table I and Table II the total energy and the charge density at $r=0$ are presented. These total energies include the normal contribution from the electron-nucleus and electron-electron interaction in the SCF procedure with the Breit and QED corrections evaluated with perturbation theory. These Breit corrections have been calculated for the average configuration and not for states coupled to good $J$-quantum numbers.

Since the early days of ionbeam-laser experiments ion beams of singly ionized $\mathrm{Ba}$ have been used in the developments of new spectroscopic techniques. These experiments have given accurate values of IS and hfs-constants. A schematic energy level diagram of the low lying states in $\mathrm{Ba}$ II constructed from the energy levels by Moore [58] is presented in Fig. 2. Some of the transitions for which high resolution laser experiments $[39,40]$ have been done are also included in this figure. In the earlier work on group IIa and IIb elements calculations were done for the $6 s_{1 / 2}, 6 p_{1 / 2}$ and $6 p_{3 / 2}$ states. Since those calculations were performed with the same nuclear parameters as for the $\mathrm{Ba}$ I configurations. In addition, calculations were done for some more states to show the effect of screening by the valence electron. A summary of the results of these DF calculations for Ba II are presented in Table III. The total energy and charge density at the nucleus are given in Column 2 and 3.

Table II. A comparison of theoretical and experimental mixing of states (in percent) for the odd parity configurations 6 s6 $p$ and $6 \mathrm{p} 5 \mathrm{~d}$. The total energy and charge density at the nucleus is given in the last columns

\begin{tabular}{|c|c|c|c|c|c|c|c|c|c|c|}
\hline $\begin{array}{l}\text { Config. } \\
6 s n p\end{array}$ & "State" & Method & $6 s n p_{1 / 2}$ & $6 s n p_{3 / 2}$ & $6 p_{1 / 2} 5 d_{3 / 2}$ & $6 p_{1 / 2} 5 d_{5 / 2}$ & $6 p_{3 / 2} 5 d_{3 / 2}$ & $6 p_{3 / 2} 5 d_{5 / 2}$ & $\begin{array}{l}\text { Total energy } \\
\text { (a.u.) }\end{array}$ & $\begin{array}{l}4 \pi|\psi(0)|_{\text {tot }}^{2} \\
\text { (a.u.) }\end{array}$ \\
\hline \multirow[t]{3}{*}{$6 s 6 p$} & ${ }^{1} P_{1}$ & $\begin{array}{l}\text { MCDF } \\
\text { LS } \\
\text { Exp }\end{array}$ & $\begin{array}{l}17.71 \\
33.33 \\
25.0\end{array}$ & $\begin{array}{l}51.76 \\
66.67 \\
75.0\end{array}$ & 11.47 & & 1.70 & 17.35 & 8129.043 & 4045756.1 \\
\hline & ${ }^{3} P_{1}$ & $\begin{array}{l}\text { DF } \\
\text { MCDF } \\
\text { LS } \\
\text { Exp }\end{array}$ & $\begin{array}{l}69.23 \\
69.07 \\
66.67 \\
75.0\end{array}$ & $\begin{array}{l}30.77 \\
25.98 \\
33.33 \\
25.0\end{array}$ & 1.29 & & 2.81 & 0.86 & $\begin{array}{l}8129.070 \\
8129.075\end{array}$ & $\begin{array}{l}4045788.1 \\
4045776.8\end{array}$ \\
\hline & ${ }^{3} P_{2}$ & $\begin{array}{l}\text { DF } \\
\text { MCDF } \\
\text { LS }\end{array}$ & & $\begin{array}{c}100 \\
95.22 \\
100\end{array}$ & 0.11 & 2.45 & 0.39 & 1.83 & $\begin{array}{l}8129.066 \\
8129.072\end{array}$ & $\begin{array}{l}4045785.9 \\
4045775.6\end{array}$ \\
\hline
\end{tabular}


Table II. Continued

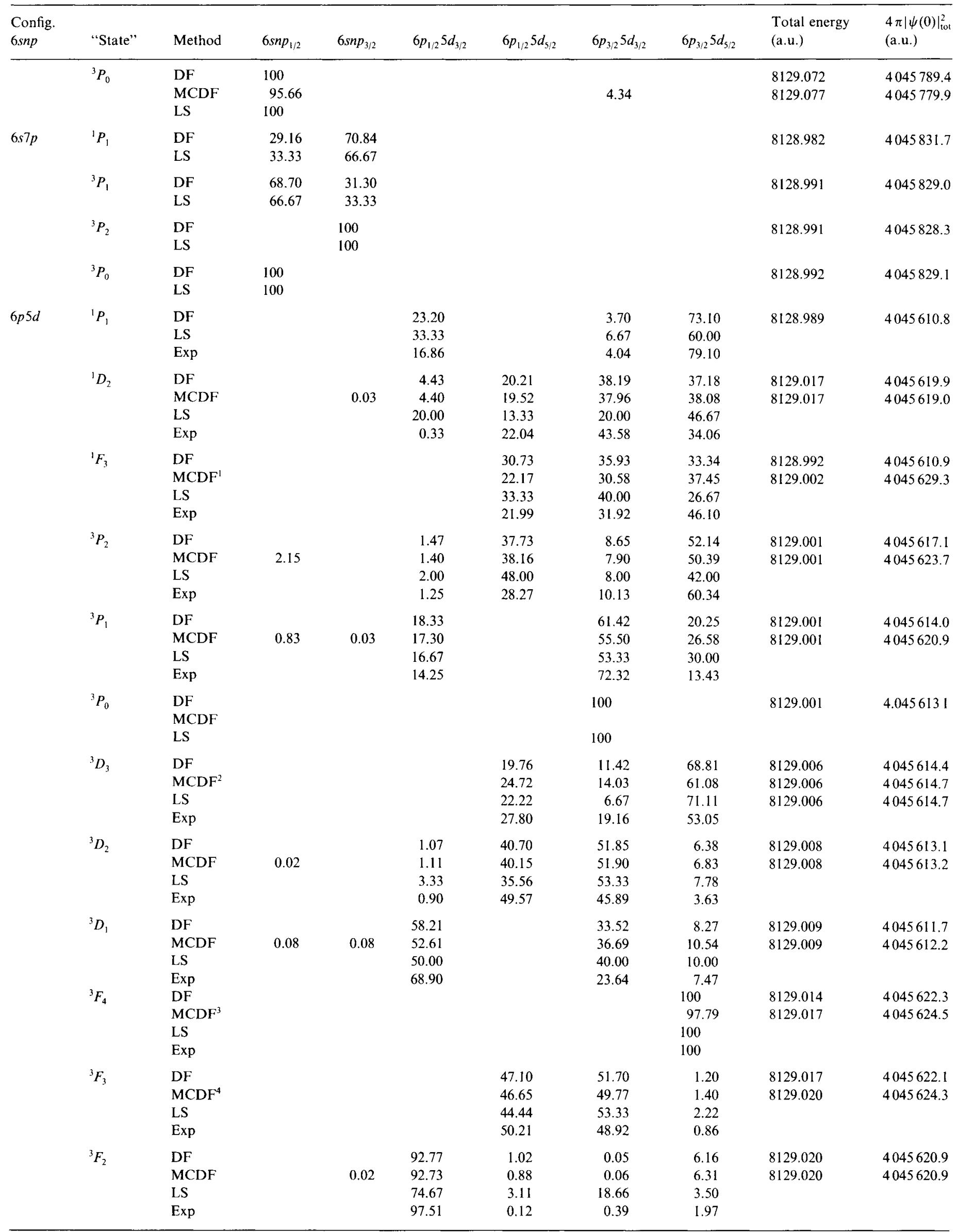

\footnotetext{
${ }^{1} 6 s_{1 / 2} 4 f_{5 / 2} 4.09 \% 6 s_{1 / 2} 4 f_{7 / 2} 5.70 \%$.

${ }^{2} 6 s_{1 / 2} 4 f_{5 / 2} 0.10 \% 6 s_{1 / 2} 4 f_{7 / 2} 0.10 \%$. $6 s_{1 / 2} 4 f_{7 / 2} 2.21 \%$

${ }^{4} 6 s_{1 / 2} 4 f_{5 / 2} 1.13 \% 6 s_{1 / 2} 4 f_{7 / 2} 1.06 \%$.
} 


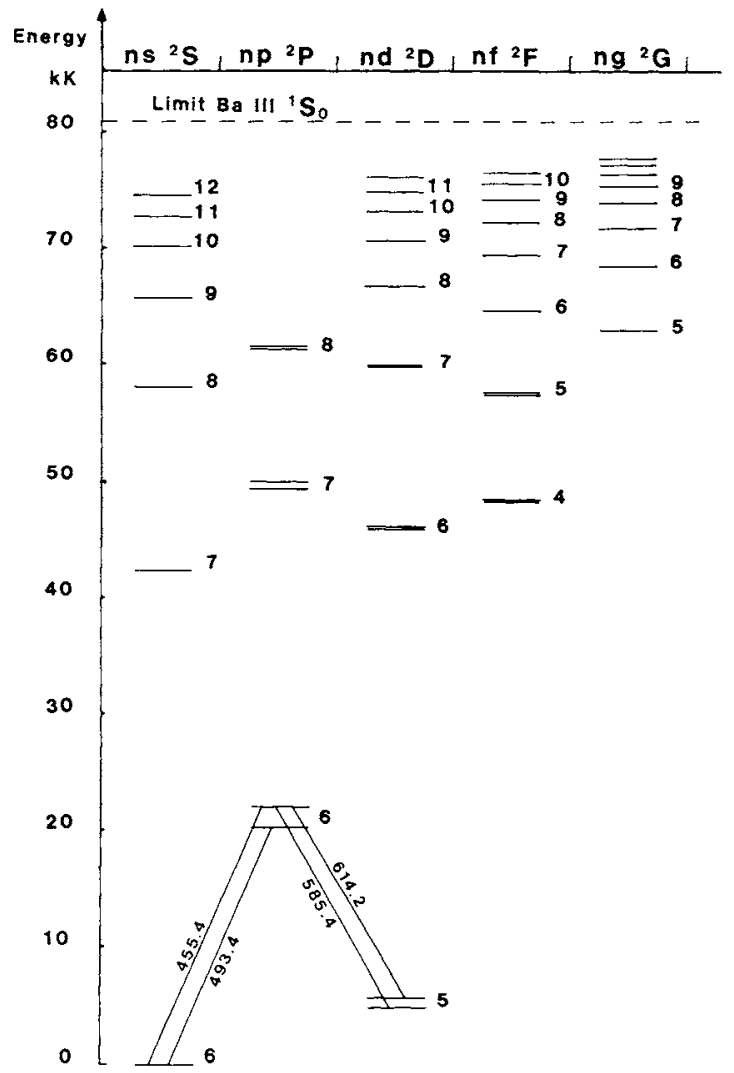

Fig. 2. Schematic energy level diagram of the lowest states in singly ionized Ba 1I. Some optical transitions are indicated and wavelengths are given in nm.

Table III. Total energies and charge densities at the nucleus for states in singly ionized $B a$ as obtained in the DF calculations. The values for the charge density are given relative to the ${ }^{2} S_{1 / 2}$ ground state

\begin{tabular}{|c|c|c|}
\hline State & $\begin{array}{l}\text { Total energy } \\
\text { (a.u.) }\end{array}$ & $\begin{array}{l}4 \pi|\psi(0)|^{2} \\
\text { (a.u.) }\end{array}$ \\
\hline $6 s_{1 / 2}$ & 8128.943 & 4045832.4 \\
\hline $7 s_{1 / 2}$ & .766 & -112.8 \\
\hline $8 s_{1 / 2}$ & .699 & -141.3 \\
\hline $9 s_{1,2}$ & .665 & -152.2 \\
\hline $10 s_{1 / 2}$ & .646 & -157.4 \\
\hline $6 p_{1,2}$ & .859 & -171.1 \\
\hline $6 p_{3 / 2}$ & .853 & -174.2 \\
\hline $7 p_{1 / 2}$ & .736 & -167.4 \\
\hline $7 p_{3 / 2}$ & .734 & -168.6 \\
\hline $8 p_{1 / 2}$ & .684 & -166.3 \\
\hline $8 p_{3 / 2}$ & .683 & -166.9 \\
\hline $9 p_{1 / 2}$ & .657 & -165.8 \\
\hline $9 p_{3 / 2}$ & .657 & -166.1 \\
\hline $10 p_{1 / 2}$ & .641 & -165.5 \\
\hline $10 p_{3 / 2}$ & .640 & -165.7 \\
\hline $5 d_{3 / 2}$ & .914 & -220.4 \\
\hline $5 d_{5 / 2}$ & .912 & -216.7 \\
\hline $6 d_{3 / 2}$ & .750 & -175.7 \\
\hline $6 d_{5 / 2}$ & .750 & -175.4 \\
\hline $7 d_{3,2}$ & .691 & -169.7 \\
\hline $7 d_{5 / 2}$ & .690 & -169.6 \\
\hline $8 d_{3 / 2}^{\prime 2}$ & .661 & -167.6 \\
\hline $8 d_{5 / 2}$ & .661 & -167.5 \\
\hline Ba III ' $S_{0}$ & .598 & -165.1 \\
\hline
\end{tabular}

\subsection{Electronic factors in the volume isotope shifts}

The total charge densities calculated for different states in $\mathrm{Ba} I$ and $\mathrm{Ba}$ II have been used for evaluation of electronic factors $F_{i}$ in a number of allowed transitions, i.e., change of parity and $\Delta L=0, \pm 1, \Delta S=0, \pm 1$ and $\Delta J=0, \pm 1$. A summary of the results is given in Table IV and $\mathrm{V}$ for transitions in $\mathrm{Ba} \mathrm{I}$ and $\mathrm{Ba} \mathrm{II}$, respectively. The first columns give the spectral notation for the transitions and the wavelength. The electronic factors $F_{i}$ are evaluated from the total charge densities at the nuclear origin $(r=0)$ as presented in Tables I to III. The effective electronic factors are $F \cdot K$ (see eq. 6 ). The values in Table III should therefore be multiplied with the factor $K=0.97$, taking into account the variation of the electronic charge density over the nuclear volume as analyzed in detail in the earlier work [31]. Since in the King plot analysis, as discussed below, the factor drops out again we did not include this correction in Table III.

A direct comparison of these electronic factors in different optical transitions with results from other works such as electronic and muonic $\mathrm{X}$-rays, is usually made through a King plot analysis. In this procedure the experimental isotope shift in one transition is related to the experimental isotope shift in another one, known as the reference transition, as described in section 2.2. The slopes of the lines as given by eq. (8) represent pure experimental quantities to be used for a direct comparison with $a b$ initio values. Theoretical slopes evaluated from the electronic factors are presented in columns 6 and 7 in Table IV. The corresponding experimental quantites calculated with $6 s^{2}{ }^{1} S_{0} \leftrightarrow 6 s 6 p^{3} P_{1}$ as the reference transition are presented in the last column. Column 4 in Table $V$ gives values for the slopes in transitions in Ba II evaluated with the transition $6 s_{1 / 2} \rightarrow 6 p_{1 / 2}$ as a reference. We notice that for almost all transitions a significant improvement is obtained with the MCDF wavefunctions compared to the single configuration DF values. In most of the cases the $a b$ initio values calculated from the MCDF values fall within the uncertainties of the experimental quantities. For Ba II good agreement is achieved with the single DF wavefunction as expected.

This King plot analysis can also be extended to a multidimensional analysis i.e., by use of many reference transitions as done by Goble and Palmer for Mo [63]. Furthermore, King plot analysis of transitions from one state to several states in another configuration opens the possibility of studying the $J$ dependence of the charge density at the nucleus in that configuration. In addition, this procedure can also be extended to compare charge densities for states in the neutral spectra with states in ionic spectra by use of isotope shift measurement in high Rydberg states for the neutral systems $[33,34]$.

Experimental charge densities at the nucleus evaluated in such a type of King plot analysis for different states in Ba I and $\mathrm{Ba}$ II are presented in Fig. 3(a). Theoretical $a b$ initio values obtained from the total charge density values at the nucleus in Table I to III are given in Fig. 3(b). Not only are the same general trends of the configuration and $J$-dependence obtained for the experimental and ab initio values, but also the agreement is nearly perfect. Of course one has to have in mind that these are relative numbers only where we have normalized just one charge density difference $6 s^{2}{ }^{1} S_{0} \rightarrow$ $6 s 6 p^{3} P_{\mathrm{I}}$ in Fig. 3(b) to the corresponding experimental value in Fig. 3(a). Relative to this charge density difference all the 
Table IV. Changes of electron densities at the nucleus for some electric dipole transitions in Ba I. These theoretical electronic factors are used for evaluation of theoretical slopes in a King-plot analysis which are compared with the experimental ones

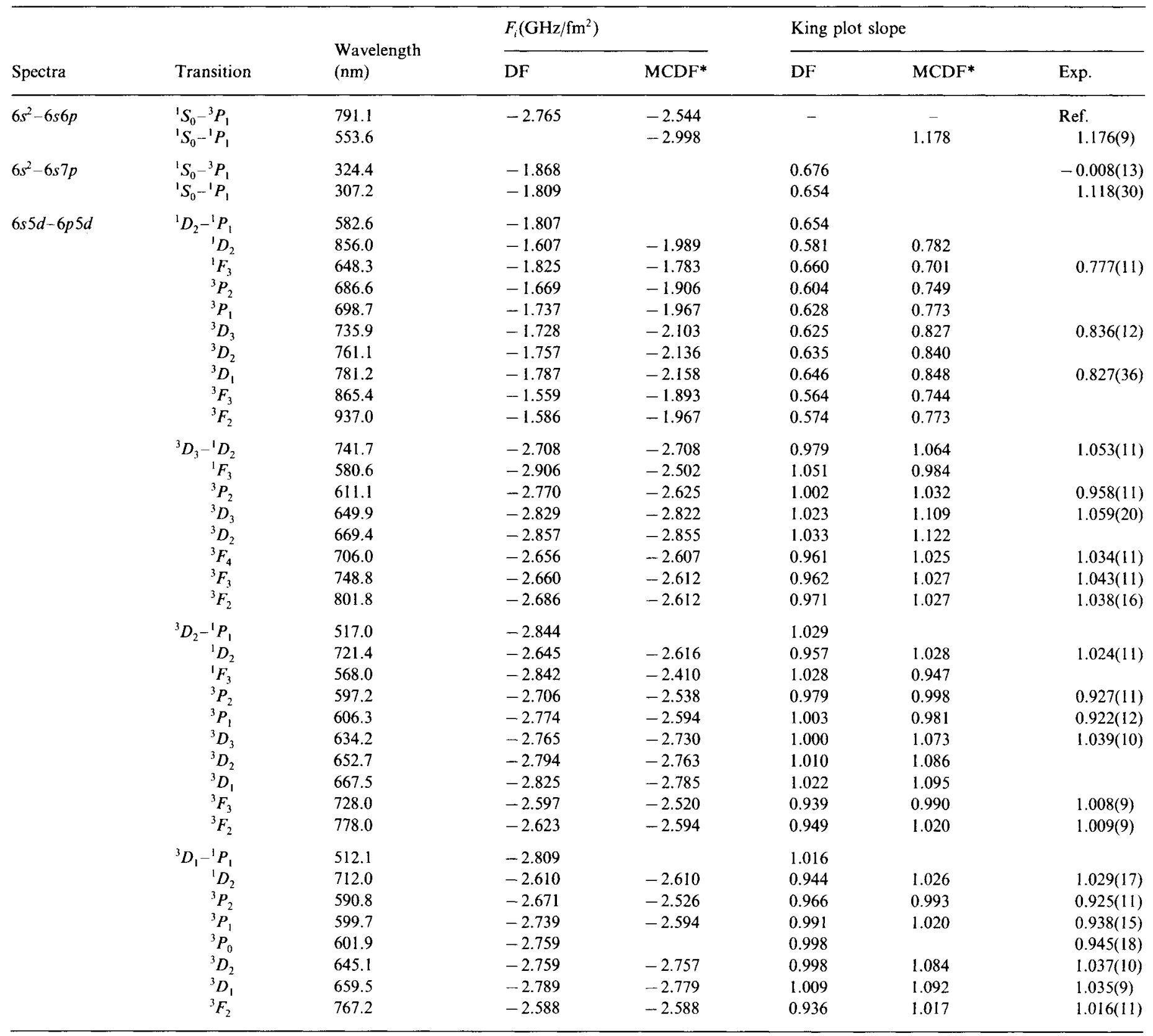

* For the $6 s 5 d^{3} D_{3}$ and ${ }^{3} D_{1}$ states the DF values are used.

other differences in the theoretical Fig. 3(b) are obtained directly from the MCDF calculations. A general trend is the relative large differences between the singlet and triplet states for nearly all of the configurations measured and calculated. As can be seen from the values in Table I and II the singletstates in the $6 s 6 p$ and $6 s 5 d$ configurations pick up a relatively large amount of configurations of $6 p$ and $5 d$ states in the MCDF procedure which has the consequence that the charge density is diminished. There are strong indications that the opposite happens in the $6 p 5 d^{\prime} P_{1}$ state as shown in [30]. However, due to not completely converged MCDF wavefunctions this result is not included.

In the presentation of the theoretical values in Fig. 3(b) we have included the charge density at the nucleus for $\mathrm{Ba}^{2+}$ i.e., the Xe-core. The addition of one $6 s$ electron which leads to $\mathrm{Ba}^{1+}$ increases the charge density by a rather large amount. However, with excitation of this electron to the $6 p$ or $5 d$ states the charge density decreases due to screening. As expected, this effect is much larger for a $5 d$ electron because the electron is in a $n=5$ shell which is much more inside the core electron cloud than the $n=6$ levels. A similar type of screening also occurs for the $6 s^{2}$ configuration in Ba I compared to the $6 s^{2} S_{1 / 2}$ state in $\mathrm{Ba}$ II. The addition of the second electron screens the other electrons by a considerable amount and its own contribution to the charge density at the nucleus finally accounts for only $35 \%$ of the total effect due to the other $6 s$ electron. Excitation of one of the $6 s$ electrons in the $6 s^{2}$ configuration to states within the $6 s 6 p$ or $6 s 5 d$ lowers the charge density at the nucleus in a way analogous to the one discussed for the $\mathrm{Ba}^{1+}$ states. Again the total effect of screening is larger for the excitation of the second electron to the $n=5$ level compared to the $n=6$ level. What is still not understood in detail is the big experimental difference in the $6 s 7 d$ excitation between the singlet and triplet states. The 


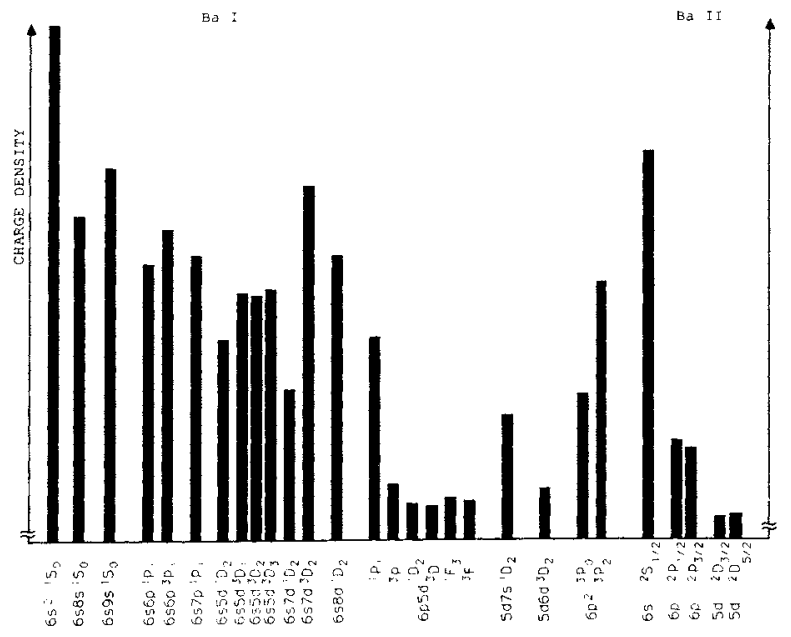

Fig. 3. (a) Experimental electron densities at the nucleus, in arbitrary units, for low lying states in Ba I and Ba II evaluated from experimental high resolution laser spectroscopy measurements, with a King plot analysis as described in the text.

$6 s 8 d^{\prime} D_{2}$ charge density again has the right trend because this Rydberg series has to end up at the $6 s$ charge density of $\mathrm{Ba}^{1+}$. Excitation of both the electrons in the $6 p 5 d$ configuration gives a value lower than that of the Xe-core, the ground configuration of $\mathrm{Ba}$ III, with the (already discussed) exception of the ${ }^{1} P_{1}$ state.

The connection of the experimental values of the charge densities for states in $\mathrm{Ba} \mathrm{I}$ and $\mathrm{Ba}$ II is based on the experimental value $\left[\varrho\left(6 s^{2}\right)-\varrho(6 s)\right] /\left[\varrho\left(6 s^{2}\right)-\varrho\left(6 s 6 p^{1} P_{1}\right)\right]=0.53(2)$ given in reference [33]. A detailed comparison of the theoretical values presented in Fig. 3(b) shows that an even better agreement is obtained if the normalization is done separately in the neutral and ionized system. Relative to the neutral, the ionized values are a little bit too low for the $6 s$ and $6 p$ states. The consistency of the calculations in the neutral system and the fact that the calculations in the ionized system are assumed to be of high quality suggests that the correction factor from reference [33] might be a bit large. But because all these numbers are only relative values it is very hard to find out where there might be an error or if the theoretical connection between the neutral and ionized system has to be improved.

In any case the agreement between experiment and theory has now reached a high quality. This means that the behav-

Table V. Comparison of different theoretical ab initio calculations of the change in the electron density at the nucleus for some transitions in Ba II. These theoretical electronic factors are used for evaluation of theoretical slopes in a King plot analysis which are compared with the corresponding experimental ones

\begin{tabular}{|c|c|c|c|c|}
\hline \multirow[b]{2}{*}{ Transition } & \multirow{2}{*}{$\begin{array}{l}\text { Wavelength } \\
(\mathrm{nm})\end{array}$} & \multirow{2}{*}{$\begin{array}{l}F_{i}\left(\mathrm{GHz} / \mathrm{fm}^{2}\right) \\
\mathrm{DF}\end{array}$} & \multicolumn{2}{|c|}{ King plot slope } \\
\hline & & & DF & Exp. \\
\hline $6 s_{1 / 2}-6 p_{1 / 2}$ & 493.4 & -3.752 & Ref. & Ref. \\
\hline $6 s_{1 / 2}-6 p_{3 / 2}$ & 455.4 & -3.820 & 1.018 & $1.026(4)^{*}$ \\
\hline $6 s_{\mathrm{I} / 2}-7 p_{\mathrm{l} / 2}$ & 202.4 & -3.671 & 0.978 & \\
\hline $6 s_{\mathrm{t} / 2}-7 p_{3 / 2}$ & 199.5 & -3.697 & 0.985 & \\
\hline $5 d_{3 / 2}-6 p_{1 / 2}$ & 649.7 & 1.081 & -0.288 & \\
\hline $5 d_{3 / 2}-6 p_{3 / 2}$ & 585.4 & 1.013 & -0.270 & $-0.244(5)^{\dagger}$ \\
\hline $5 d_{5 / 2}-6 p_{3 / 2}$ & 614.2 & 0.932 & -0.248 & $-0.235(5)^{\dagger}$ \\
\hline
\end{tabular}

* Wendt et al. [39].

* Van Hove et al. [40].

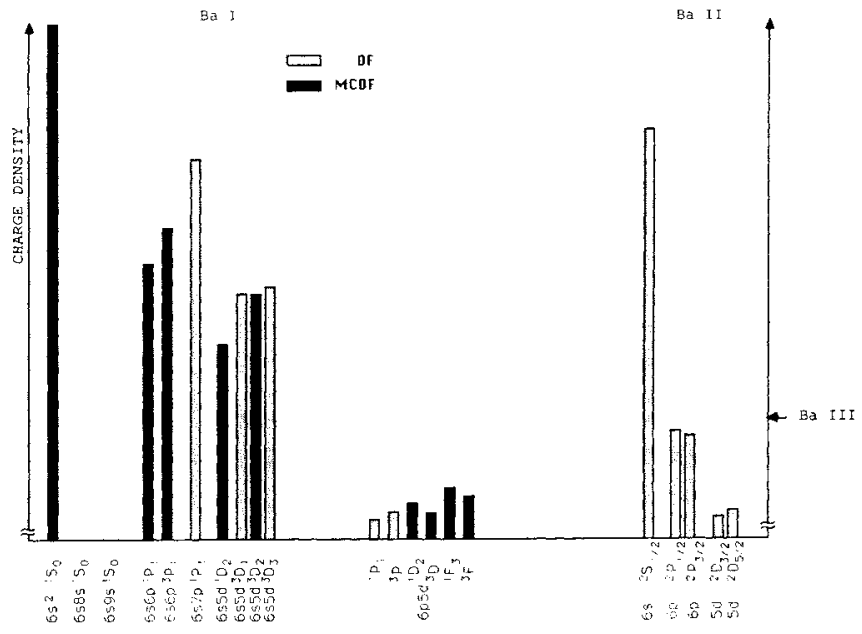

Fig. 3. (b) Theoretical electron densities at the nucleus for low lying states in Ba I, Ba II and the value for the ground state of Ba III marked with an arrow in the figure. The values for $\mathrm{Ba} \mathrm{II}$ and $\mathrm{Ba}$ III have been evaluated from single Dirac-Fock (DF) wavefunctions while those for $\mathrm{Ba} I$ have been calculated from MCDF (dark) or DF (light) wavefunctions as described in the text. The scale of this figure has been chosen so that the charge density difference $6 s^{2}{ }^{1} S_{0} \rightarrow 6 s 6 p{ }^{1} P_{1}$ is the same as in Fig. 3(a).

iour of the wavefunctions within the small region of the extended nucleus on the order of $6 \mathrm{fm}$ can be discussed and probed in a reliable way.

Absolute values of the electronic factors in optical transitions can be drived from optical isotope shift data and known $\delta\left\langle r^{2}\right\rangle^{A A^{\prime}}$ values. The $\delta\left\langle r^{2}\right\rangle$ values can be obtained from the analysis of muonic isotope shifts. The $F_{i}$ value for the $6 s^{2}{ }^{1} S_{0} \rightarrow 6 s 6 p{ }^{1} P_{1}$ transition evaluated in this way by Shera et al. [53] was found to be $-3.04(26) \mathrm{GHz} / \mathrm{fm}^{2}$, while Kunold et al. [64] obtained a value of $F_{\mathrm{j}}=-3.99(65)$ $\mathrm{GHz} / \mathrm{fm}^{2}$. The reason for this discrepancy is not clear although it should be noted that the $\left\langle r^{2}\right\rangle^{A A^{\prime}}$ values used by Shera cover a bigger region of $\delta\left\langle r^{2}\right\rangle^{A A^{\prime}}$ values and should therefore determine the $F_{i}$ value better.

\subsection{Hyperfine structure constants}

Analysis of the experimentally determined hfs-constants for heavy elements have mostly been done with a relativistic effective one-body operator acting on non-relativistic $L S$ coupled states as introduced by Sandars and Beck [63] and reviewed in an earlier work [16]. The non-relativistic $L S$ coupled states in such an approach are usually given as intermediate wavefunctions determined by a least-square fit of parametrized energy expressions including the electrostatic and magnetic interactions between the electrons to a chosen order. Using these wavefunctions, hyperfine structure constants can be given as a linear combination of hyperfine parameters, in general three parameters for the magnetic dipole and three for the electric quadrupole interaction for each open shell. Experimental values of these parameters are then usually determined by a least-square fit of the parametrized hyperfine structure constants to the experimental ones. Such a procedure works reasonably well for systems where there is little electron-electron correlation and/or in particular, valence-valence correlation. Effects of the last kind can be treated by additional effective two-body operators $[11,17,66,67]$. This approach was used by Grundevik et al. [35] to estimate the mixing effects of the contact hfs on the ${ }^{3} P$ and ' $P$ states from $n s n^{\prime} p$ configurations. 
Table VI. Comparison of experimental magnetic dipole ( $A$ ) and electric quadrupole (B) interaction constants in $\mathrm{MHz}$ for ${ }^{137} \mathrm{Ba}$ with different theoretical calculations. The electric quadrupole moment is taken to be $0.34 \mathrm{~b}$

\begin{tabular}{|c|c|c|c|c|c|c|c|}
\hline \multirow[b]{2}{*}{ Config. } & \multirow[b]{2}{*}{ State } & \multicolumn{3}{|c|}{ A-constant } & \multicolumn{3}{|c|}{ B-constant } \\
\hline & & DF & MCDF & Exp. & DF & $\mathrm{MCDF}$ & Exp. \\
\hline \multirow[t]{11}{*}{$6 p 5 d$} & ${ }^{\prime} P_{1}$ & 102.2 & & $-11.6(2.0)^{\mathrm{a}}$ & 15.6 & & $11.3(2.0)^{\mathrm{a}}$ \\
\hline & ${ }^{\prime} D_{2}$ & 54.6 & 55.0 & $78.7(3)^{\mathrm{b}}$ & -13.2 & -13.8 & $0.6(1.0)^{\mathrm{b}}$ \\
\hline & ${ }^{\prime} F_{3}$ & 60.6 & 53.0 & $67.3(2)^{\mathrm{a}}$ & 26.5 & I4.3 & $86.0(1.0)^{\mathrm{a}}$ \\
\hline & ${ }^{3} P_{2}$ & 41.1 & 68.6 & $11.3(3)^{\mathrm{a}}$ & 38.9 & 39.3 & $21.5(9)^{\mathrm{a}}$ \\
\hline & ${ }^{3} P_{1}$ & 82.7 & 142.5 & $125.2(3)^{\mathrm{a}}$ & 3.0 & -1.2 & $-16.4(5)^{\mathrm{a}}$ \\
\hline & ${ }^{3} D_{3}$ & 59.9 & 62.6 & $9.0(4)^{\mathrm{a}}$ & 78.3 & 83.6 & $-16.4(1.0)^{\mathrm{a}}$ \\
\hline & ${ }^{3} \mathrm{D}_{2}$ & 61.4 & 61.9 & $9.9(2)^{\mathrm{a}}$ & 14.2 & 14.1 & $-1.2(3)^{\mathrm{a}}$ \\
\hline & ${ }^{3} D_{1}$ & 106.5 & 86.0 & $172.9(3)^{\mathrm{b}}$ & -6.3 & 4.6 & $-3.5(0.6)^{b}$ \\
\hline & ${ }^{3} F_{4}$ & 42.0 & 45.4 & $23.5(3)^{6}$ & 92.9 & 92.8 & $111.0(1.3)^{\mathrm{b}}$ \\
\hline & ${ }^{3} F_{3}$ & 77.4 & 77.7 & $87.4(3)^{b}$ & 59.1 & 57.1 & $83.0(1.4)^{\mathrm{b}}$ \\
\hline & ${ }^{3} F_{2}$ & 144.7 & 144.8 & $218.0(3)^{\mathrm{h}}$ & 37.5 & 37.7 & $45.3(1.0)^{\mathrm{b}}$ \\
\hline \multirow[t]{4}{*}{$6 s 5 d$} & ${ }^{\prime} D_{2}$ & 47.7 & -13.4 & $-82.180(3)^{\mathrm{c}}$ & 32.7 & 40.8 & $59.564(14)^{\mathrm{c}}$ \\
\hline & ${ }^{3} D_{3}$ & 369.1 & & $456.559(4)^{\mathrm{d}}$ & 46.7 & & $47.390(16)^{\mathrm{d}}$ \\
\hline & ${ }^{3} \mathrm{D}_{2}$ & 260.4 & 320.2 & $415.928(3)^{\mathrm{d}}$ & 48.5 & 47.9 & $25.899(13)^{\circ}$ \\
\hline & ${ }^{3} D_{1}$ & -367.8 & & $-520.536(3)^{\mathrm{d}}$ & 18.5 & & $17.890(3)^{\mathrm{d}}$ \\
\hline \multirow[t]{3}{*}{$6 s 6 p$} & ${ }^{1} P_{1}$ & & -53.4 & $-109.2(1.2)^{\mathrm{e}}$ & & -7.7 & $51(12)^{e}$ \\
\hline & ${ }^{3} P_{2}$ & 621.4 & 580.2 & & 60.6 & 61.4 & \\
\hline & ${ }^{3} P_{1}$ & 790.5 & 804.0 & $1150.59(2)^{\mathrm{f}}$ & 54.9 & 52.5 & $41.61(2)^{f}$ \\
\hline \multirow[t]{3}{*}{$6 s 7 p$} & ${ }^{\prime} P_{1}$ & -82.8 & & $-105.3(4)^{g}$ & -2.8 & & $16.6(6)^{g}$ \\
\hline & ${ }^{3} P_{2}$ & 754.8 & & & 10.6 & & \\
\hline & ${ }^{3} P_{1}$ & 822.8 & & & 9.3 & & \\
\hline
\end{tabular}

a Grundevik et al. Ref. [36].

${ }^{b}$ Grundevik et al. Ref. [35].

' Schmelling, S. G., Phys. Rev. A9, 1097 (1974).

' Gustavsson, M., Olsson, G. and Rosén, A., Z. Phys A290, 231 (1979).

e Kluge, H.-J. and Sauter, H., Z. Phys. 270, 295 (1974).

i zu Putlitz, G., Ann. Phys. 11, 248 (1963).

g Eliel, et al. Ref. [37].

As a result, two additional two-body parameters appear along with the six parameters originating from the one-body operator for the $6 p$ and $5 d$ electrons. Comparison of experimentally determined hyperfine parameters converted to radial integrals, showed a reasonable agreement for the orbital part with $a b$ initio radial integrals for the $6 p$ and $5 d$ electrons evaluated from DF wavefunctions calculated for the average energy of the specific configuration.

In our approach to the analysis of the hfs-constants the angular coupled wavefunctions or intermediate wavefunctions are given by the expansion coefficients of the MCDF wavefunctions as presented in Tables I and II. The hfs constants are then evaluated from these wavefunctions according to eq. (16) which means that these hfs-constants are purely ab initio.

A summary of the results and a comparison with experimental hfs constants is presented in Table VI. The first and second columns present the configuration and $S L J$ states, respectively. Our $a b$ initio values evaluated with the single DF and MCDF wavefunctions are presented in the following columns with a comparison of the experimental hfs-constants. For some of the states we have not been able to achieve completely converged MCDF wavefunctions and there are therefore a few states missing. The agreement with the experimental hfs-constants is not particularly good for many of the states. One of the reasons is probably that our wavefunctions do not include configurations with excitations from the core. Such configurations have been found to be important in nonrelativistic MBPT calculations [17, 28, 29]. On the other hand our calculations are expected to include accurately and on equal footing the effect of relativity and valence-valence correlation for the electrons in the open shells.

\section{Conclusion and outlook}

In the preceeding part of this paper we have made an extensive analysis of quantities like term energies, differences of charge densities at the nucleus between different terms (i.e., $F$ factors) as well as magnetic dipole and electric quadrupole hyperfine structure constants of $\mathrm{Ba} I$. The reason for doing this comparison between experiment and theory was to answer the question of the quality of the MCDF method. Because all these experimental quantities test different parts of the wavefunctions the quality of the agreement is very different. The term energies depend on the wavefunctions at all distances, while the $a(\gamma J)$ and $b(\gamma J)$ factors in the hyperfine structure mainly depend on the inner part of the wavefunctions because the integrals involved contain $r^{-2}$ and $r^{-3}$ integration factors and the volume isotope shift depends only on the wavefunction within the nucleus.

What is clear from the comparison between theory and experiment is the fact that the multiconfiguration calculations present an improvement compared with the single configuration ones. The best example is the strong contribution from some states within the $6 p 5 d$ configuration which have a large mixing of the states with $J=1^{-}$in the $6 s 6 p$ configuration. Of course it is clear that all the higher energetic states with the same angular momentum and parity will probably also have 
further influences. But the relative good agreement with experiment, which shows that all the energies are more or less correct and the relative order is correct too, indicates that the contribution due to higher states is expected to be similar for all the states (including the ground state) so that all differences remain largely unchanged.

The best agreement between experiment and theory can be seen in the evaluation of the $F$ factors. Fig. 3 shows a nearly perfect agreement of the trends and dependence of the different states in the considered configurations. As the $F$-factors depend on the charge density at the nucleus, this demonstrates that at least the $s_{1 / 2}$ and $p_{1 / 2}$ wavefunction contributions are more or less correct. Of course there may be an absolute error in the calculations but the non-inclusion of higher $s_{1 / 2}$ and $p_{1 / 2}$ wavefunctions is expected to have an effect similar to that considered in the discussion of the term energies. Therefore, this part drops out in the calculation of the differences.

The agreement is very much different for the calculation of the $a(\gamma J)$ and $b(\gamma J)$ factors in the hyperfine structure splitting where all parts of the wavefunctions contribute in a relative large inner region. Of course, the inclusion of more configurations increases the agreement but the results remain very far off the experimental results. This is a clear indication that a good multiconfiguration calculation has to include many more configurations, especially of core excitations, than have been included in this type of relativistic MCDF calculation. The MBPT approach is much more effective for these quantities but is also much more complicated.

In conclusion, one may say that the relativistic MCDF wavefunctions for a heavy element like $\mathrm{Ba}$ leads to relatively good agreement for the energy levels as well as to accurate prediction of the volume isotope shift but is still far off in the prediction of accurate $a(\gamma J)$ and $b(\gamma J)$ factors in hyperfine structure splittings.

\section{Acknowledgement}

This work was financially supported in part by the Swedish Natural Science Research Council (NFR), and the Gesellschaft für Schwerionenforschung Darmstadt.

\section{References}

1. Articles in Proceedings from the "International Conference on Laser Spectroscopy" III, IV, V, VI, VII (Springer Series in Optical Sciences. Vol. 7, 21, 30, 40, 49, 55).

2. Articles in Proceedings from the "International Conference on Atomic Physics", 1972, 1974, 1976, 1978, 1980, 1982 (Plenum Press New York and London).

3. Articles in "Progress in Atomic Spectroscopy" (Edited by W. Hanle and H. Kleinpoppen, Plenum Press (1978, 1979, 1984).

4. Kopfermann, H., "Nuclear moments" (Trans. E. E. Schneider) Academic Press, New York (1958).

5. Brix, P. and Kopfermann, H., Rev. Mod. Phys. 30517 (1958).

6. Shirley, D. A., Rev. Mod. Phys. 36339 (1964).

7. Kuhn, H. G., "Atomic Spectra" Longmans, London (1969).

8. Heilig, K. and Steudel, A., Atomic Data and Nuclear Data Tables 14, 613 (1974).

9. Bauche, J. and Champeau, R.-J., Adv. At. Mol. Phys. 12, 39 (1976).

10. King, W. H., "Isotope Shifts in Atomic Spectra" (Plenum Press, New York (1984).

11. Armstrong, Jr, L., "Theory of Hyperfine Structure of Free Atoms", Wiley-Interscience, New York (1971).

12. Condon, E. U. and Shortley, G. H., "The Theory of Atomic Spectra",
Cambridge University Press $(1935,1963)$.

13. Slater, J. C., "Quantum Theory of Atomic Structure", Vol. I and II McGraw-Hill, New York (1960).

14. Froese-Fischer, C., "The Hartree-Fock Method for Atoms", Wiley \& Sons, New York (1977).

15. Grant, I. P., Proc. Roy. Soc. London A262, 555 (1961).

16. Lindgren, I. and Rosén, A., "Case Studies in Atomic Physics" 4, 93 (1974).

17. Lindgren, I. and Morrison, J., "Atomic-Many Body Theory", Springer Series in Chemical Physics, Vol. 13, Berlin, Heidelberg, New York, Springer (1982).

18. Lindgren, I. and Lundqvist, S., Eds., Physica Scripta 2, 227 (1980).

19. Froese-Fischer, C., Comp. Phys. Comm. 1, 151 (1969); 4, 107 (1972); 14, 145 (1978).

20. Desclaux, J. P., Comp. Phys. Comm. 9, 31 (1975).

21. Grant, I. P., McKenzie, B. J., Norrington, P. H., Mayers, D. F. and Pyper, N. C., Comp. Phys. Comm. 21, 207 (1980).

22. Trees, R. E., Phys. Rev. 83, 756 (1951); 84, 1089 (1951); 85, 382 (1952).

23. Seaton, M. I., Proc. Phys. Soc. London 88, 815 (1966).

24. Lu, K. T. and Fano, U., Phys. Rev. A2, 81 (1970); Lu, K. T., Phys. Rev. A4, 579 (1971).

25. Aymar, M., Physics Reports 110, 163 (1984).

26. Froese-Fischer, C. F. and Hansen, J. E., Phys. Rev. A24, 631 (1984).

27. Matthias, E., Rinneberg, H., Beigang, R., Timmermann, A., Neukammer, J. and Lücke, K., Atomic Physics 8, Proceedings of the "Eighth International Conference on Atomic Physics", Gothenburg, Aug. 1982; (Edited by I. Lindgren, A. Rosén and S. Svanberg), Plenum Press, New York and London 1983. This article gives a summary of results for the alkaline earth elements.

28. Olsson, G. and Salomonson, S., Z. Physik A307, 99 (1982).

29. Salomonson, S., Z. Physik A316, 135 (1984).

30. Fricke, B., Grundevik, P., Lindgren, I., Olsson, G., Olsson, T., Rosén, A. and Torbohm, G., Phys. Lett. 97A, 183 (1983).

31. Torbohm, G., Fricke, B. and Rosén, A., Phys. Rev. A31, 2038 (1985).

32. Rosén, A., Fricke, B. and Torbohm, G., Z. Physik, A316, 157 (1984).

33. Hogervorst, W. and Eliel, E. R., Z. Phys. A310, 19 (1983).

34. Hogervorst, W. and Eliel, E. R., Phys. Rev. A27, 2995 (1983).

35. Grundevik, P., Gustavsson, M., Olsson, G. and Olsson, T., Z. Phys, A312, 1 (1983).

36. Grundevik, P., Lundberg, H., Nilsson, L. and Olsson, G., Z. Phys. A306, 195 (1982).

37. Eliel, E. R., Hogervorst, W., Olsson, T. and Pendrill, L. R., Z. Phys. A311, 1 (1983).

38. Jitschin, W. and Meisel, G., Z. Phys. A295, 37 (1980)

39. Wendt, K., Ahmad, S. A., Buchinger, F., Mueller, A. C., Neugart, R. and Otten, E. W., Z. Phys. A318, 125 (1984).

40. van Hove, M., Borghs, G., De Bisschop, P. and Silverans, R. E., J. Phys. B15, 1805 (1982).

41. Olsson, T., Jönsson, G. and Robertsson, L., to be published.

42. Reviews are given in "Proceedings of the Workshop on Foundations of Relativistic Theory of Atomic Structure" ANL-Report ANL-80-126 (1981).

43. Reviews are given in "Relativistic Effects in Atoms, Molecules and Solids" (Edited by G. L. Malli) Plenum Press, New York and London (1983).

44. Breit, G., Phys. Rev. 34, 553 (1929).

45. Mann, J. P. and Johnson, W. R., Phys. Rev. A3, 1267 (1971).

46. Fricke, B., Desclaux, J. P. and Waber, J. T., Phys. Rev. Lett. 28, 714 (1972).

47. Cheng, K. T., Desclaux, J. P. and Kim, Y. K., J. Phys. B11, L359 (1978).

48. Desclaux, J. P., in Ref. [43].

49. McKenzie, B. J., Grant, I. P. and Norrington, P. H., Comp. Phys. Comm. 21, 233 (1980).

50. Mohr, P., in Ref. [42].

51. Mohr, P., pp. 145-167 in Ref. [43].

52. Landolt-Börnstein; Zahlenwerte und Funktionen, Bd.2 (Edited by Hellwege, K. H.) Springer Verlag, Berlin (1967).

53. Shera, E. B., Wohlfart, H. D., Hoehn, M. V. and Tanaka, Y., Phys. Lett. 112B, 124 (1982).

54. Myers, W. D., "Droplet Model of Atomic Nuclei" Plenum, New York (1977).

55. King, W. H., J. Opt. Soc. Am. 53, 638 (1963).

56. Schwartz, C., Phys. Rev. 97, 380 (1955). 
57. Bohr, A. and Weisskopf, V. F., Phys. Rev. 77, 94 (1950).

58. Moore, C. E., "Atomic Energy Leveis", Vol. I, II, III; NBS Circ. 467, Washington D.C. 1949, 1952, 1958.

59. Verges, J., Unpublished material 1984. Private communication.

60. Camus, P., Dieulin, M. and El Himdy, A., Phys. Rev. A26, 379 (1982).

61. Vassen, W., Bente, E. and Hogervorst, W., J. Phys. B20, 2383 (1987).

62. Olsson, G., Thesis, Dept. of Physics, University of Göteborg (1982).
63. Goble, A. T. and Palmer, C. W. P., J. Phys. B18, 2181 (1985).

64. Kunold, W., Schneider, M., Simons, L. M., Wüst, and Abela, R., Z. Phys. A312, 11 (1983).

65. Sandars, P. G. H. and Beck, J., Proc. R. Soc. (London), A289, 97 (1965).

66. Bauche-Arnoult, C., Proc. R. Soc. (London) A322, 361 (1971).

67. Dembczynski, J., Journal de Physique 41, 109 (1980). 